\title{
Optimized Balance of Plant for a Medium-size PEM electrolyzer. Design, Modelling and Control
}

\author{
Julio José Caparrós ${ }^{1 *}$, Francisco José Vivas ${ }^{1}$, Francisca Segura ${ }^{1}$, José Manuel Andújar ${ }^{1}$ \\ ${ }^{1}$ Centro de Investigación en Tecnología, Energía y Sostenibilidad - CITES (Research Center for Technology, \\ Energy, and Sustainability), University of Huelva, Campus El Carmen, 21071 Huelva, Spain \\ *julio.caparros@diesia.uhu.es
}

SNE 31(3), 2021, 133-141, DOI: 10.11128/sne.31.tn.10574 Received: March 10, 2021 (Selected EUROSIM 2019 Postconf. Publ.); Revised: August 31, 2021; Accepted: September 3, 2021 SNE - Simulation Notes Europe, ARGESIM Publisher Vienna ISSN Print 2305-9974, Online 2306-0271, www.sne-journal.org

Abstract. This paper presents the design, modelling and control simulation of the Balance of Plant (BoP) for a medium-size PEM (Proton Exchange Membrane) electrolyzer. Taking into consideration the main chemical process that occurs in the electrolysis of a PEM electrolyzer, the BoP must be clearly divided into two main parts: water management and hydrogen production. An exhaustive control system is developed to test the working conditions that will allow the PEM electrolyzer to generate hydrogen in a safe and efficient way. The aim of this study is to find an equilibrated solution between minimal BoP and a correct performance, always into safety conditions of hydrogen generation. Automation software is used to model the system and perform a control logic, according to the desirable operation of the PEM electrolyzer. The BoP developed, along with the electrolyzer model and the control logic proposed contribute the PEM electrolysis technology to be expanded in the industrial market and the hydrogen to be widely used as energy carrier, as part of the renewable energy systems.

\section{Introduction}

More and more governments, companies and general society are committed to a change in energy policies, always oriented towards more energy efficient actions and more renewable energy use. But renewable energy technologies present a high dependence on climatic factors and they have not yet achieve enough reduced cost to compete with conventional supply energy systems [1]. Additionally, most of renewable sources-based systems implement their energy storage capacity in battery-based solutions.
But the use of battery presents some limitations as the relation size-energy density and the useful lifetime due to charging-discharging cycles. Then, the use of hybrid power systems based on hydrogen as backup is presented as an ideal solution to fill the gaps in the energy supply that the different renewable energy sources may provoke [2]. Studies have proven their key influence in improving the operation of hybrid energy systems and their application in smart grids [3].

Regarding the hydrogen production technology, nowadays electrolysis as a sustainable method to produce hydrogen from water, as long as the electricity required comes from a renewable energy source is the option most used in real systems [4]. Therefore, an electrolyser can convert electrical energy into chemical energy, with the use of hydrogen as an energy vector [3]. Within the types of DC electrolysis, according to the nature of the electrolyte, it is possible differentiate between Alkaline electrolysis and PEM (Proton Exchange Membrane) electrolysis.

Alkaline technology uses liquid electrolyte, with high concentrations of potassium hydroxide to provide ionic conductivity and to participate in the electrochemical reactions. By cons, PEM technology replaces the liquid electrolyte by a solid polymer electrolyte, which selectively conducts positive ions such as protons. The protons participate in the water-splitting reaction instead of hydroxide, creating a locally acidic environment in the cell [5].In alkaline electrolysis, Figure 1, the two electrodes are immersed in an electrolyte, which is usually a $\mathrm{KOH}$ or $\mathrm{NaOH}$ solution [6]. In this way, an alkaline environment is achieved, where the electrical resistivity of the water will be reduced, making the process easier. The DC voltage to be applied must be greater than that required for the water splitting and thus cause the faradaic current [7]. The electrodes are separated by a diaphragm, which allows to keep the gases separated, while being permeable to water and the passage of hydroxide ions. 


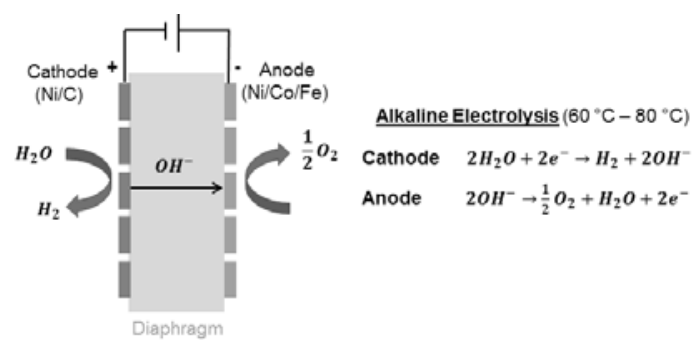

Figure 1: Alkaline electrolytic cell.

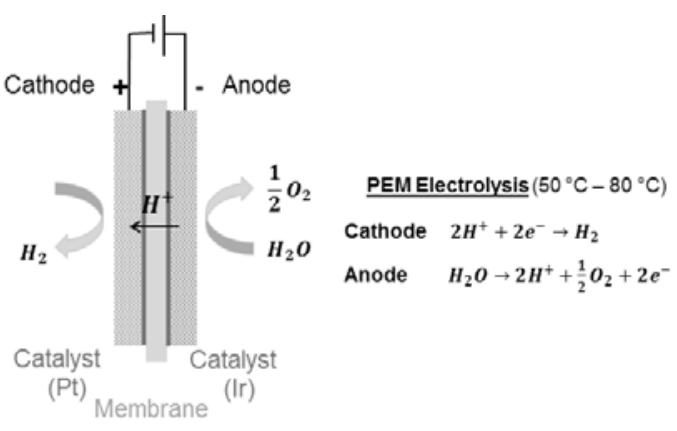

Figure 2: PEM electrolytic cell.

On the other hand, in PEM electrolysis, Figure 2, the two electrodes are in contact with the electrolyte which is a solid polymer, usually Nafion. Bipolar plates are also typically added between the solid electrolyte and the electrodes, made of platinum for the cathode and iridium for the anode, with the aim of adding resistance to corrosion [8], produced during the uncontrolled polarity of the cells and fluctuating charges.

Comparing both technologies, the main disadvantages of alkaline technology are the limited current density ( $0.4 \mathrm{Acm}-2)$, low partial power range, limited by the inertia of the liquid electrolyte (higher than 20\%$40 \%$ ) [9], crossover of gases and low operating pressure (< 10-15 bar) [10]. By contrast, PEM electrolyzers can reach a current density of up to $2 \mathrm{Acm}-2$, the polymer electrolyte membrane guarantees a low gas crossover, allowing the PEM electrolyzers to work under a lower partial load range (0-10\%), and it can have a compact design. This allows obtaining high enough operating pressures (30-40 bar) [11] to directly fill hydrogen tanks [5].

Additionally, regarding alkaline technology, the $\mathrm{KOH}$ electrolyte is highly corrosive in comparison with the PEM technology. However, poisoning by foreign ions appears and thus it has to be highly considered. The water can be easily contaminated by the impurities it contains, as well as by the corrosion produced in the metallic components of the system, such as the water pipes or even the stack components themselves.
This poisoning will result in an increase in the cell cathodic overvoltage and a reduction in operating performance [12], in addition to affecting the membrane in a reduction of its proton conductivity. Then, these are the reasons why an exhaustive design and control of the BoP (involving water management, conductivity, and purity) is important to make PEM electrolysis technology will become a competitive hydrogen production option [10].

This paper contributes to hydrogen technology implantation into the energy industrial sector. Additionally, although previous studies have been conducted in the simulation of PEM electrolyzers as power-hardware-inloop (PHIL) simulator [13], dSPACE Hardware-in-theLoop simulators [14], multiphysics simulators [15], dynamic simulators based on MATLAB [16] and mathematical dynamic Simulink simulators [17], it is hard to find papers that use software tools based on totally integrated automation logic.

This work combines the modelling of the control design, necessary for the safe and effective performance of the system, with the simulation of its parameters, providing compatibility and a simulation environment and quality testing. This paper describes the design, modelling and control simulation of the BoP for a medium-size PEM electrolyzer. In Section 1 the BoP is described, differentiating the hydrogen subsystem from the water subsystem. Next, Section 2 shows the control logic developed to control the electrolysis plant. Modelling and control simulation results obtained by automation software are presented in Section 3 and Conclusions from Section 4.

\section{Balance of Plant of a PEM Electrolyzer}

In this section it is provided a solution for the implementation of the BoP, within the specifications of safety and performance. With hydrogen technologies, it is important to carry out a study of the equipment in subsystems that capture in the BoP, which has been shown to be essential to achieve a reliable design with optimal production [18]. As it has been have seen previously, in PEM technology it is very important to ensure specific water conditions at time that safe hydrogen production conditions, in order to guarantee the correct functioning of the electrolyzer. Therefore, a specific water treatment and control system is proposed, Figure 3. This system starts acquiring water from a distilled water tank, which is convenient to have low conductivity, to ensure a longer life of the electrolytic stack. 
Once the water has been introduced into the system, an injection pump is used to ensure an adequate input flow into the system. After passing through the injector pump, the water is passed through a two-phase filtering system to give it a low conductivity. Otherwise, the PEM stack could be critically impaired. In the first phase, it is obtained a Type II conductivity (ASTM Standards for Laboratory Reagent Water (ASTM D1193-91), (<1 $\left.\mu \mathrm{Scm}^{-1}\right)$, and in the second phase, the conductivity level achieves Type I conductivity $\left(<0.056 \mu \mathrm{Scm}^{-1}\right)$.

Next the filtering stage, the water is introduced into a tank that has a double function: 1) to be a buffer with the aim to adjust the water flow inside the circuit, and 2) to act as a sink that collects the water obtained in the different drying stages of the hydrogen produced by the PEM stack. From the water level control tank, the water continues its flow to the water-control and recirculation phase. The recirculation pump regulates the water flow after the water level control tank, and the sensors' block is used by the controller to have information of all critical water parameters, such as temperature, pressure, flow, conductivity and purity, before being injected into the PEM stack. The recirculation line is proposed as a means to correct the conductivity of the water, in case it is not within the allowed range.

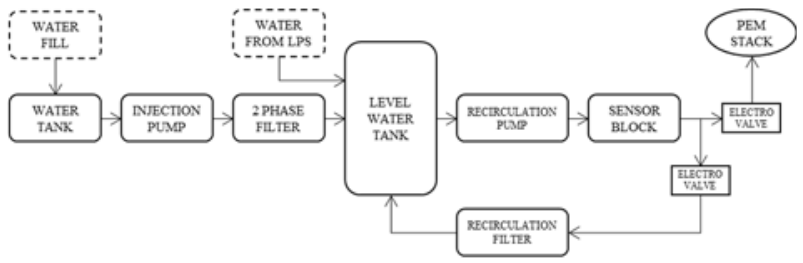

Figure 3: Balance of plant (BOP) of a PEM electrolytic stack: Water subsystem.

Additionally to the water subsystem, the hydrogen subsystem must be thoroughly designed to guarantee the safety parameters, as well as the correct drying, in order to eliminate the humidity that may be contained, sending it back to the water level tank.

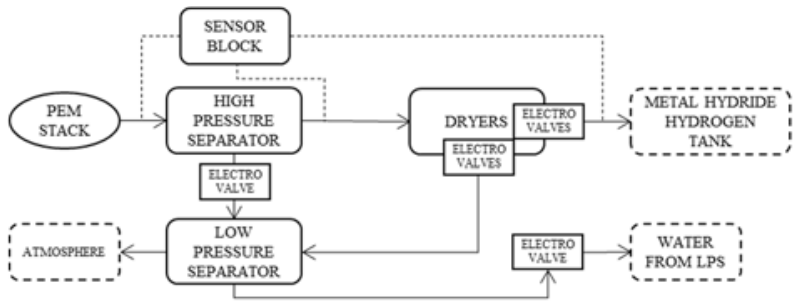

Figure 4: Balance of plant (BOP) of a PEM electrolytic stack: Hydrogen subsystem.
The hydrogen subsystem, Figure 4, is made up the PEM stack, followed by a high pressure separator. The separator takes advantage of the pressure difference in water contained in the form of moisture to dry the hydrogen. Once a high humidity gradient is reached in the high pressure separator, it allows the wet hydrogen flow (dirty hydrogen) to enter into the low pressure separator. Here, the hydrogen that can be mixed into the atmosphere is released, and the water is sent to the level water tank (water subsystem, Figure 3). By contrast, the dry hydrogen (clean hydrogen) from the high pressure separator, continues to the dryers' stage. Here, a pressure swing adsorption drying stage (PSA) is proposed, which stores and expels the water in three drying cycles. The released water is sent to the low pressure separator, following the same process previously described. After the drying phase, he hydrogen produced will be stored in metal hydride tanks. Throughout the process of hydrogen production, there is a sensors' block, which serves to control the pressure and temperature parameters of hydrogen flow in its different production and drying stages, prior to final storage.

\section{Design of the Control System}

The control system to be implemented into the PEM electrolyzer should be able to have information and act accordingly into the two main parts of the BoP: water management subsystem and hydrogen production subsystem. Additionally, the control logic must include the sequence of the operating states, the cooling stage, the nitrogen inertization stage, the power supply control and the management of the warnings and alarms generated during the electrolyzer operation.

\subsection{Water management subsystem}

Regarding the water management subsystem, Figure 5, the control logic includes three main parts: water level control block, conductivity control block and physical variables measurement and control block. Then, regarding the first control block, the activation of the level sensor in the water level tank, it activates the injection pump when the system requires more water, having a mid level. When the level is high enough, the pump is deactivated. In case of lowering the level too much, at a low level, the PEM electrolyzer stops.

In relation to the conductivity control block, the water conductivity is regulated by acting over an electrovalve to put in work the recirculation line. 
Then, when the conductivity is low, the water is supplied directly to the stack without the need to subject the water to more purification treatment. In case the conductivity is medium (Type I < conductivity < Type II), before production, the recirculation circuit will be open to recirculate the water back to the purification filter. If this occurs during production, a warning is activated. And finally, if the conductivity rises above type II, the electrolyzer will be kept stopped, an alarm will be triggered, and through a process of disconnection and inertization, and an alarm will be triggered.

The physical parameters as temperature, pressure and water flow are watched with the aim to guarantee the system performance inside its operating specifications, useful life and safe conditions Otherwise, the system stops.

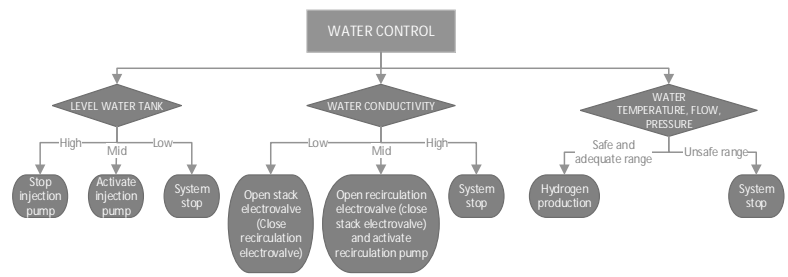

Figure 5: PEM stack control flow diagram; control diagram of the water management subsystem.

\subsection{Hydrogen production subsystem}

For the hydrogen subsystem control, Figure 6, it is necessary to detect the water level both in the high pressure separator (HPS) and the low pressure separator (LPS). When a mid level is detected in HPS, the valve will be open to let the accumulated water passing towards the LPS. When this happens, and the level drops, the valve closes again. In case of a high level is detected, the electrolyzer stops. Level control in LPS works in a similar way, allowing the water to pass to the water level tank when it has enough water accumulated into the LPS, as long as the valve to LPS, from HPS, is closed. In an electrolysis process, it is crucial to avoid direct contact between the water and hydrogen lines. If the level in LPS is low, the valve closes since there is not enough water to transport.

After de HPS and LPS, the dryers of the PSA stage follow a three-phase cyclic process during production. This is defined temporarily with the opening and closing of electrovalves that allow the hydrogen flow to the final storage, the accumulation of water and the purge of the water accumulated through the LPS. During all the process, temperature and pressure are controlled, entering the system in stop if they are outside the established range.

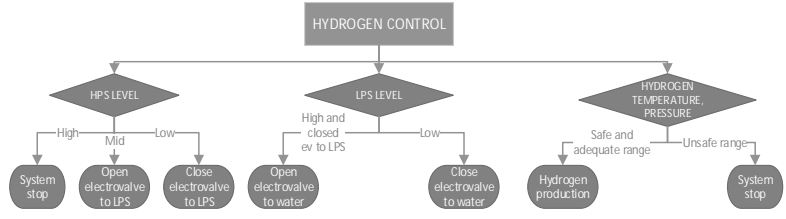

Figure 6: PEM stack control flow diagram; Hydrogen control diagram.

\section{Testing of the Control Logic. Simulation Results}

In this section, there will be presented the results that show the proper operation of the system following the sequence established by the developed control logic. The hardware-software tool used to implement the control logic is based on Siemens ${ }^{\circledR}$ SIMATIC STEP 7 Basic V15 platform, with TIA Portal tools for modelling, in addition to PLCSIM and WinCC RT for simulation.

Then, all the processes involved into the electrolysis plant are visualized trough the main screen, PEM electrolyzer management monitor, Figure 7a. This monitor shows the operating state of the system, allows to activate, stop or pause the production as well as to start a nitrogen inerting process. Additionally, this monitor shows operating conditions of the water and hydrogen subsystems, and it allows the cooling subsystem operation and the alarm management.

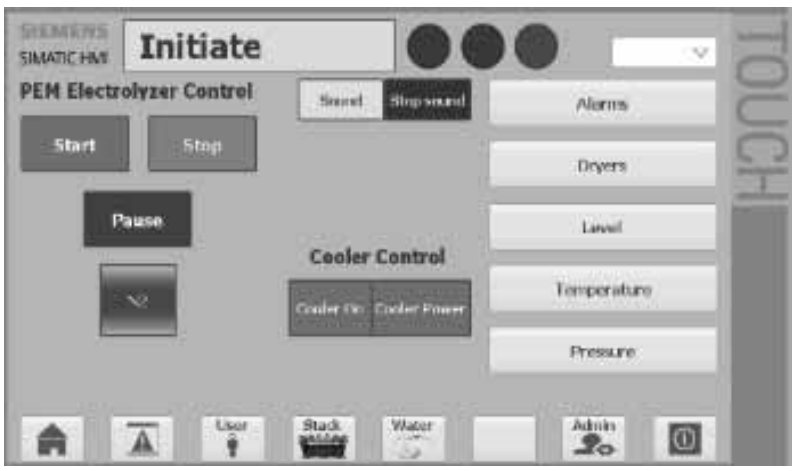

Figure 7a: Screen 1: PEM Electrolyzer management monitor.

The control system also has an interface for the stack parameters, Figure $7 b$, through which the simulation of the current and voltage values can be monitored, in addition to the power graph in real time.

The simulation of the hydrogen drying process, Figure $7 \mathrm{c}$, shows in real time the status of each of the dryers, process status and remaining time. 


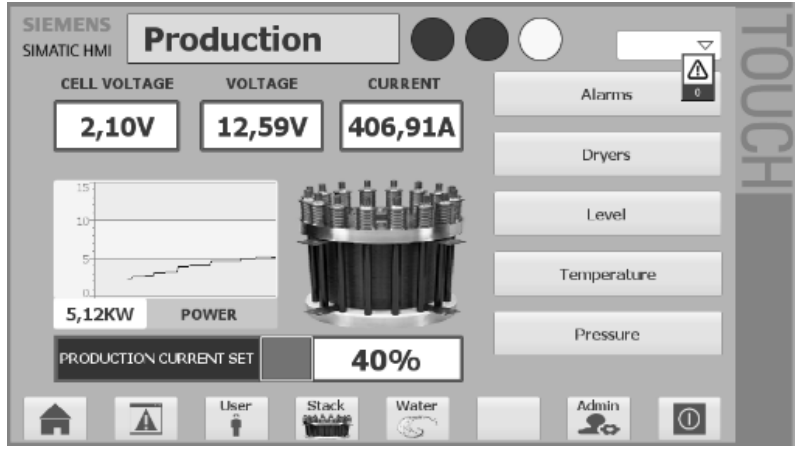

Figure 7b: Stack interface.

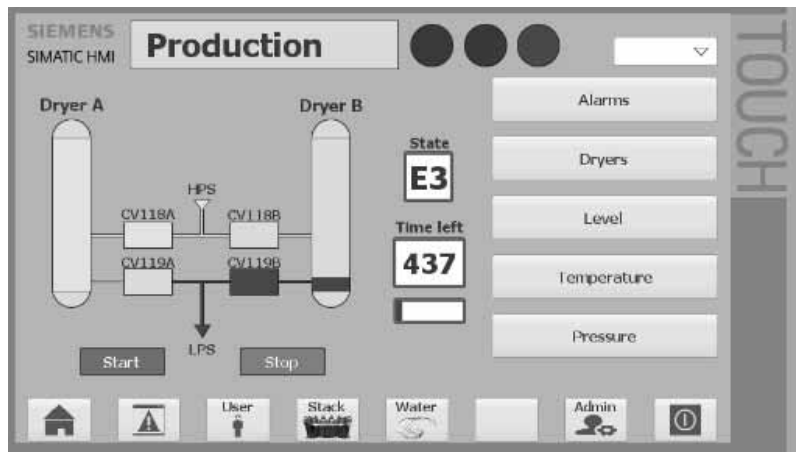

Figure 7c: Dryers stage interface.

\subsection{Simulation test of water subsystem control}

In this case, regarding the scheme of the balance of plant presented in Figure 3 and the control diagram developed in Figure 5, the test will be used to check the proper operation of the water management subsystem. Then, the system starts from a low level of water, Figure 8a. This involves the activation of the injection pump until the water level rises the higher level allowed, Figure $8 \mathrm{~b}-\mathrm{Fi}$ gure $8 \mathrm{c}$. After this, the water pump will be disconnected and the water level decreases; no water is injected into the line while the stack is consuming it for the hydrogen production, Figure 8d.

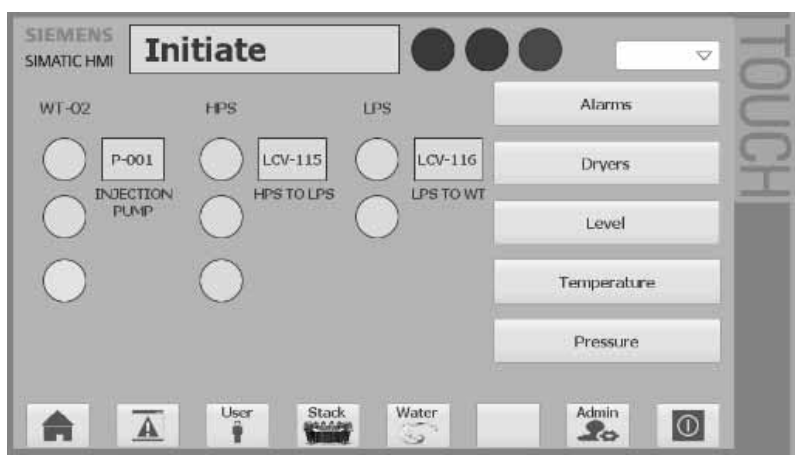

Figure 8a: Water subsystem. Low level of water; increasing.

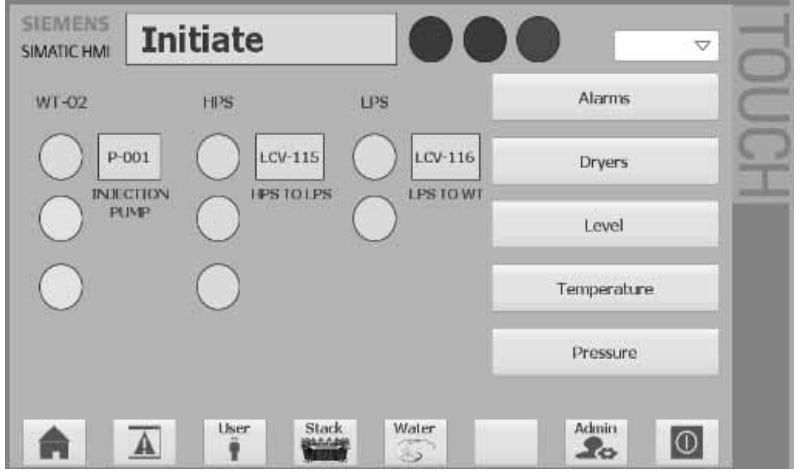

Figure 8b: Water subsystem. Mid level of water; increasing.

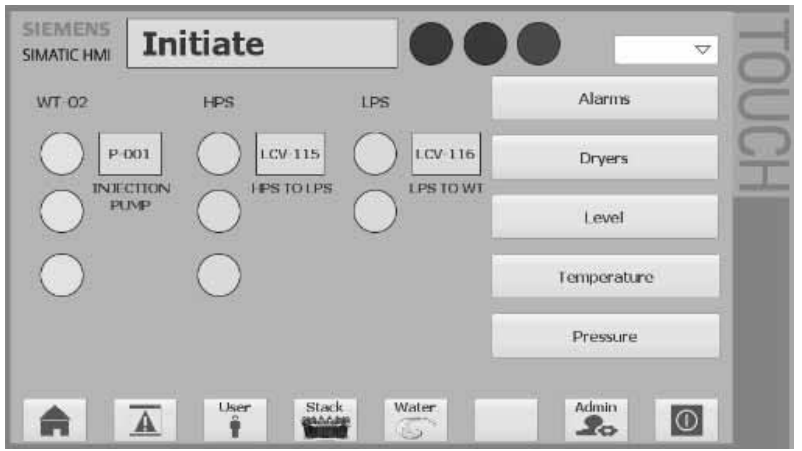

Figure 8c: Water subsystem. High level of water.

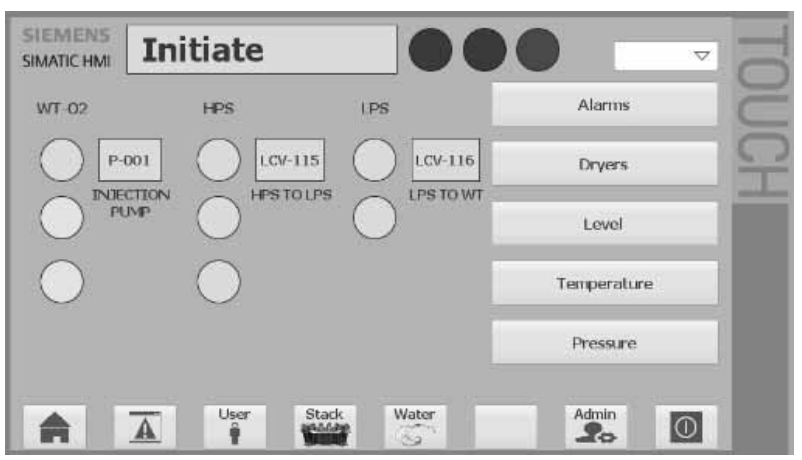

Figure 8d: Water subsystem. Mid level of water; decreasing.

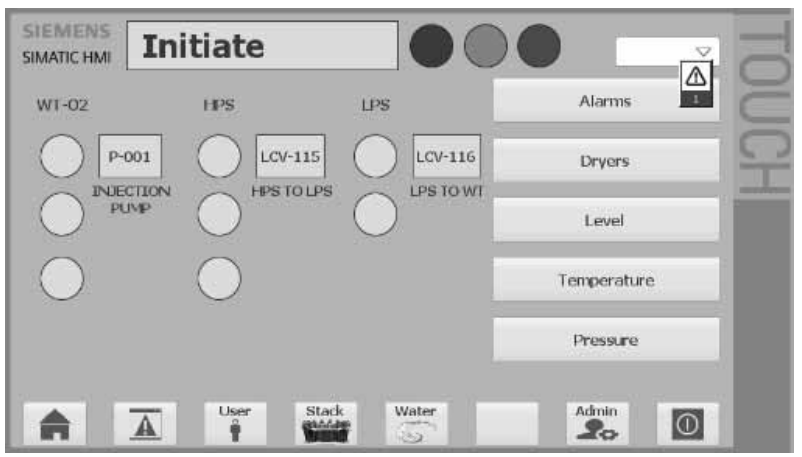

Figure 8e: Water subsystem Alarm: low level of water. 
As the injection pump is deactivated, the water level begins to decrease until returning to the low level when it will be reactivated. A red colour led warms that the level has drop completely, Figure 8e.

Additionally, in case an error measurement due to a sensor malfunctioning, a yellow-colour warning light advise about this, Figure $8 f$.

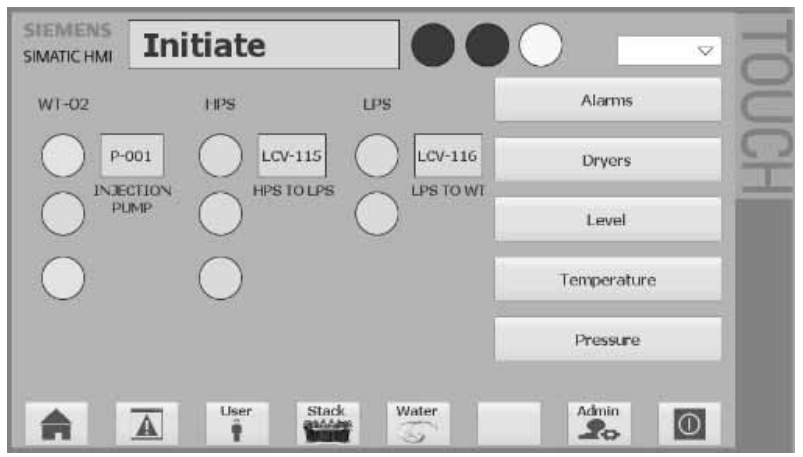

Figure 8f: Water subsystem Warning: failure of level sensor measurement.

On the other hand, from the main monitor interface it is possible to show if the water flow meets with the water conductivity and temperature requirements, Figure 9a. Then, in case Type I < conductivity < Type II, a warming led is activated, Figure 9b, and it will turn into an alarm and the system stop (inertization stage) when the water conductivity rises Type II, Figure 9c.

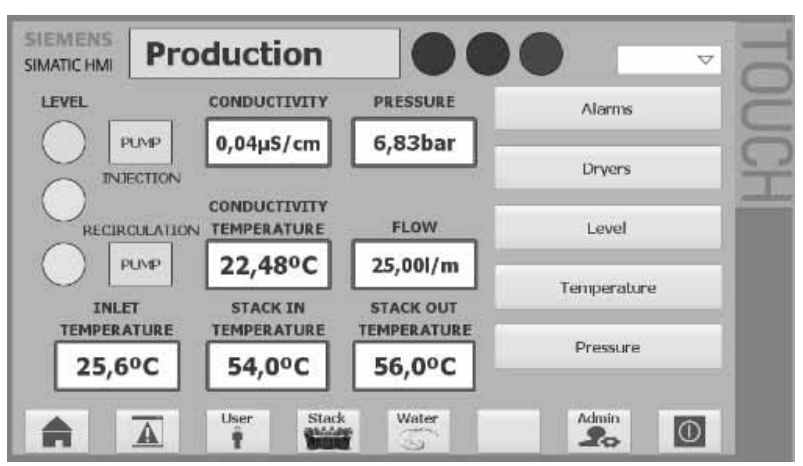

Figure 9a: Water management monitor.

In the same way, the control logic is responsible for controlling the water temperature, Figure 10a. Then, when the water temperature increases above the higher allowed value $\left(68^{\circ} \mathrm{C}\right)$, the warning led is activated and the cooler is put into operation, Figure 10b.

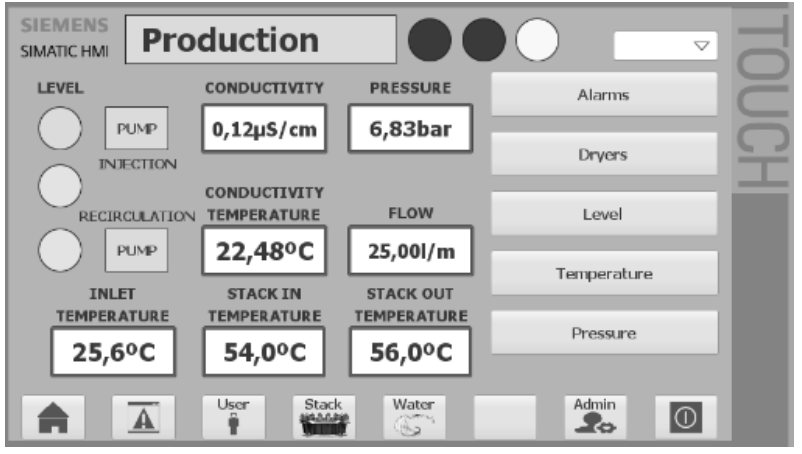

Figure 9b: Water subsystem. Warning: middle conductivity value.

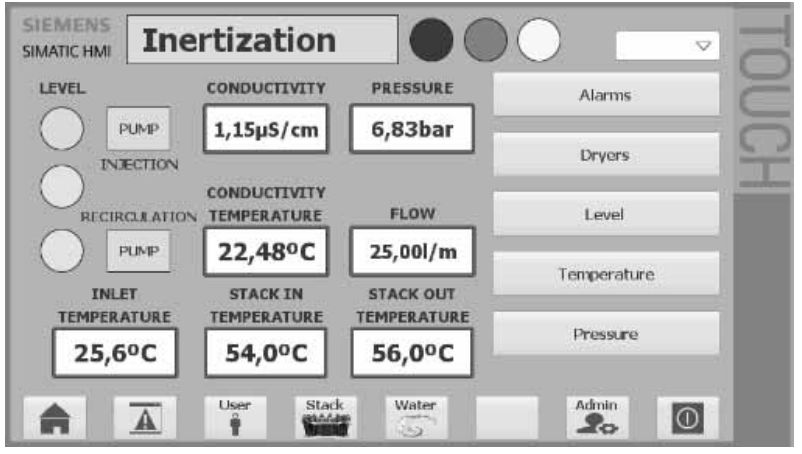

Figure 9c: Water subsystem. Alarm and inertization: high conductivity value.

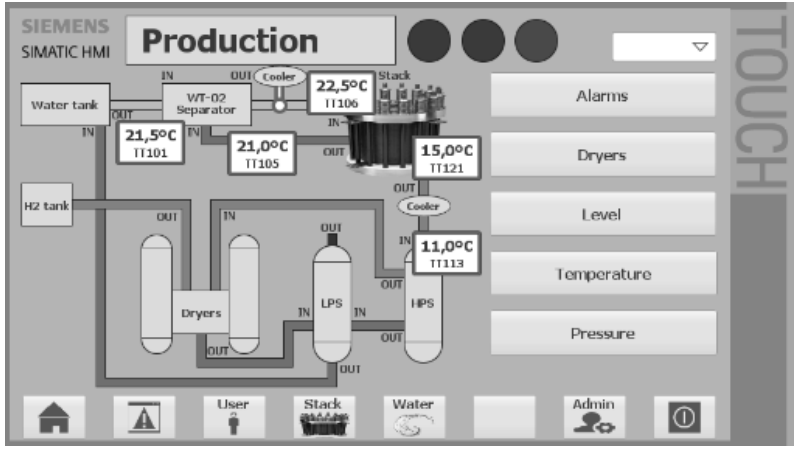

Figure 10a: Water subsystem. Temperature interface.

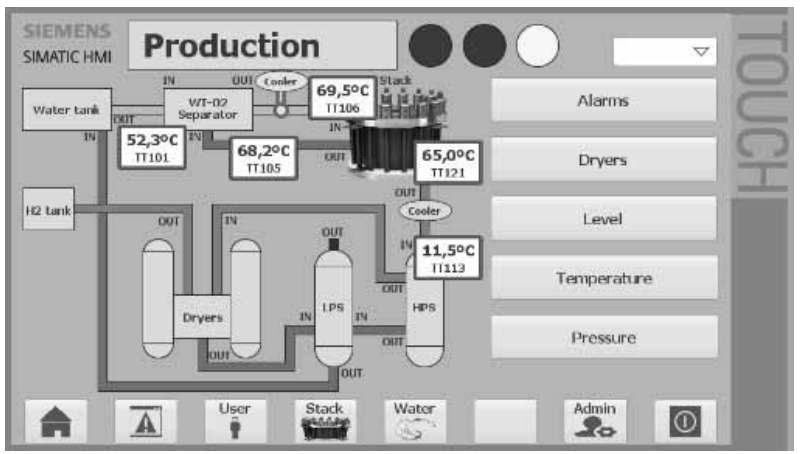

Figure 10b: Water subsystem. Warning: high water temperature. 


\subsection{Simulation test of hydrogen subsystem control}

This second test will show the behaviour of the hydrogen production subsystem according to the balance of plant shown in Figure 4 and the logic control designed in Figure 6. Then, the test start considering a low level of condensates in HPS, Fig Figure 11a, and it will rise. When it reaches the medium level, the valve is opened, Figure $11 b$.

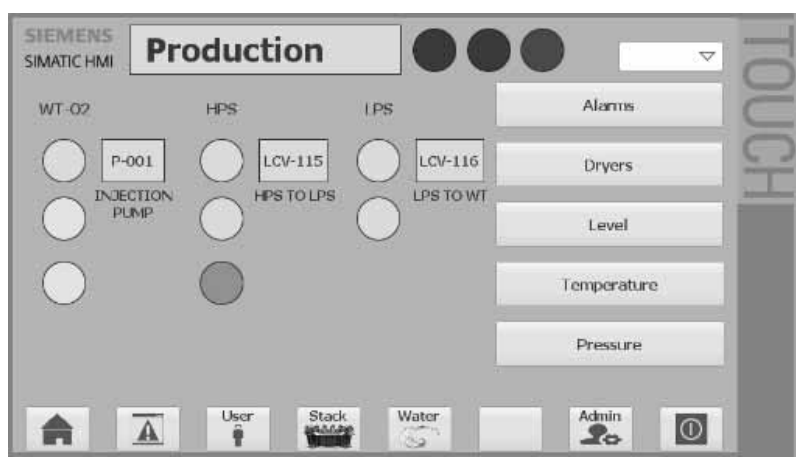

Figure 11a: Hydrogen subsystem. Low level in HPS; increasing.

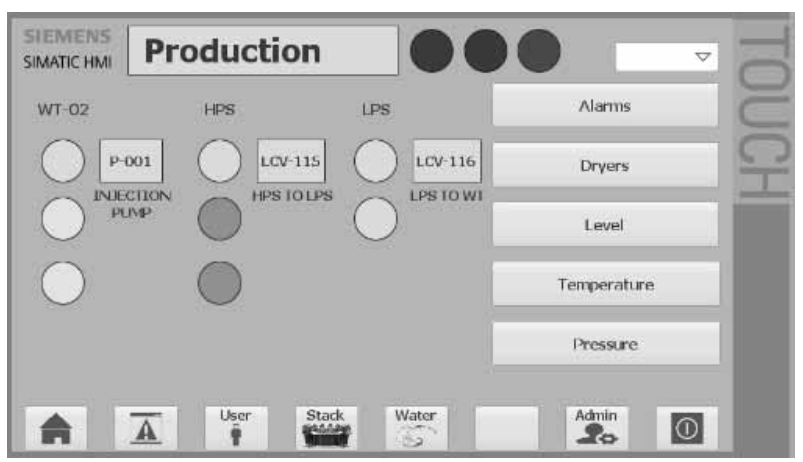

Figure 11b: Hydrogen subsystem. Mid level in HPS; valve opening.

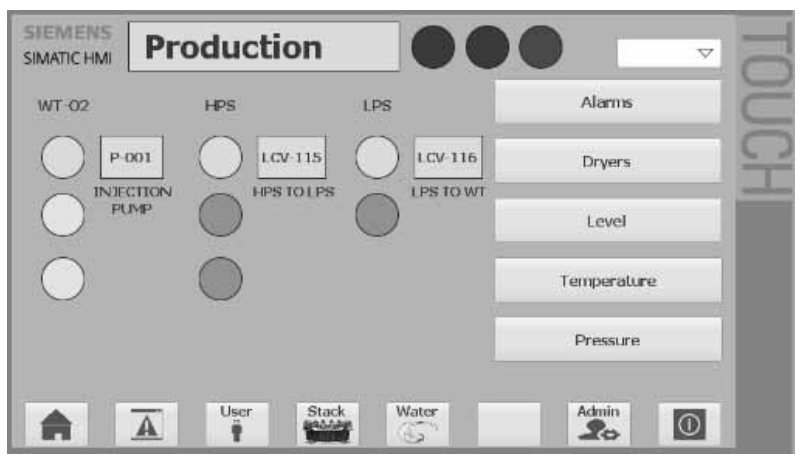

Figure 11c: Hydrogen subsystem. Low level in LPS; increasing.
Then, the condensates start their passage towards LPS, Figure 11c, until reaching the high level of LPS, Figure 11d. At this moment, it is necessary to wait for the level to drop in HPS, and with this the valve of the step to LPS, so that the opening of the LPS outlet valve is allowed, Figure 11e.

In case the HPS achieves its highest level, an alarm is activated and the production is stopped, Figure $11 \mathrm{f}$.

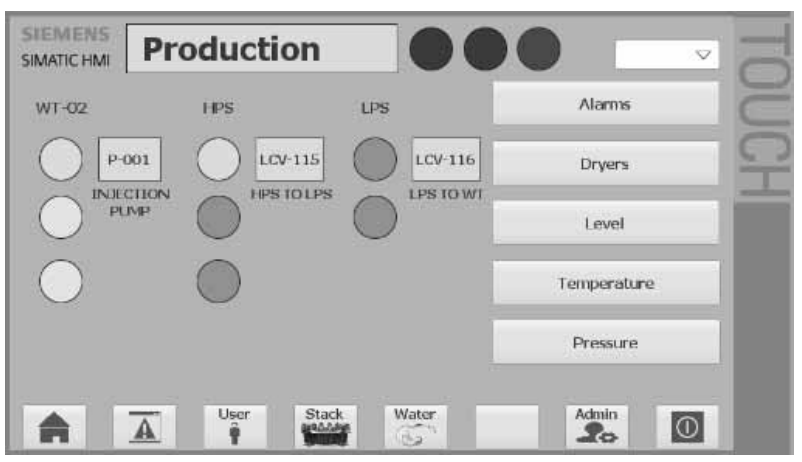

Figure 11d: Hydrogen subsystem. High level in LPS; closed valve.

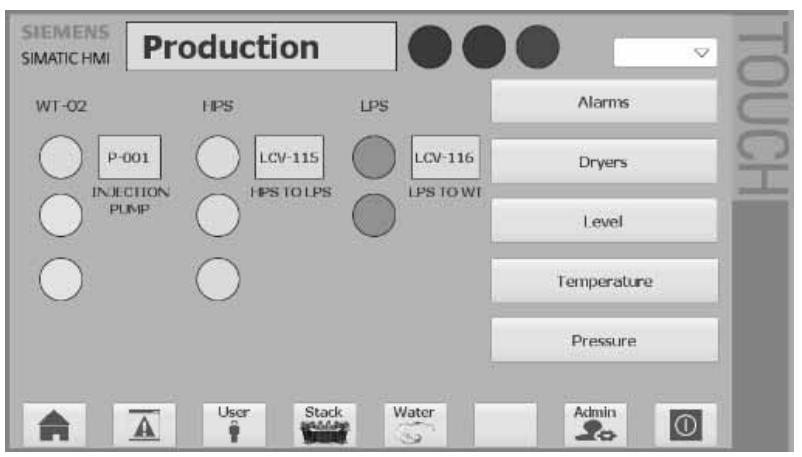

Figure 11e: Hydrogen subsystem. High level in LPS; open valve.

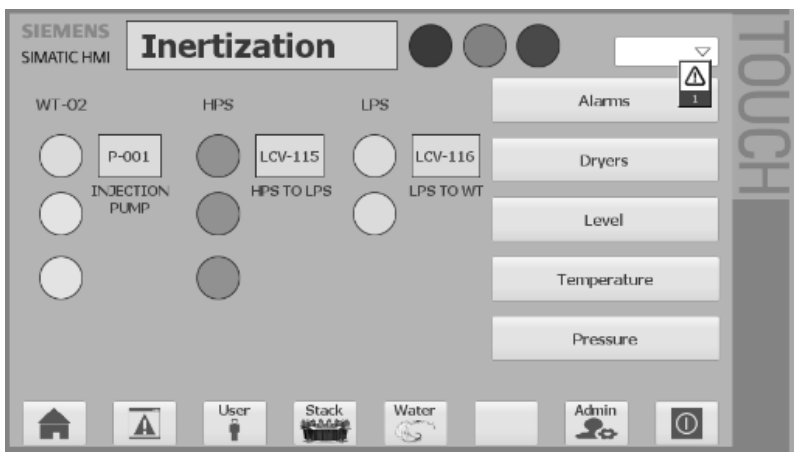

Figure 11f: Hydrogen subsystem. Alarm: high level in HPS. 
Similar to water management test, it is possible to check the temperature of the hydrogen flow. In case the hydrogen temperature is above the highest allowed value $\left(72^{\circ} \mathrm{C}\right)$, a warning will advise us, Figure $12 \mathrm{a}$ and if it continues increasing $\left(75^{\circ} \mathrm{C}\right)$, an alarm is activated and the system stops; it pass from production to inertization state, Figure $12 b$.

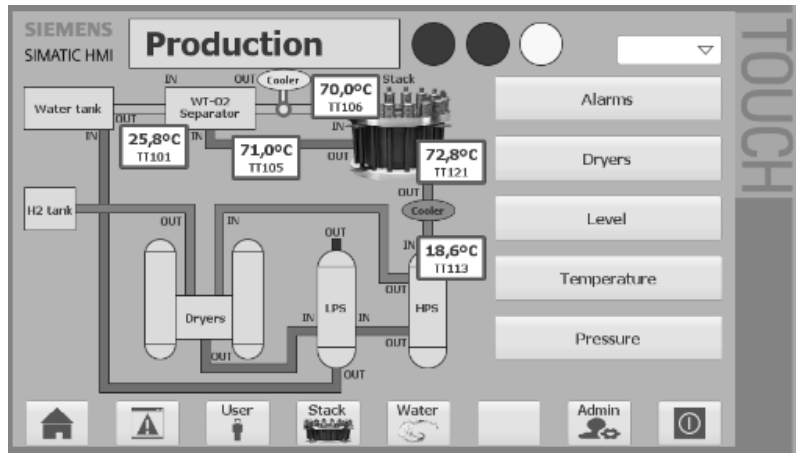

Figure 12a: Hydrogen subsystem. Warning in the temperature interface.

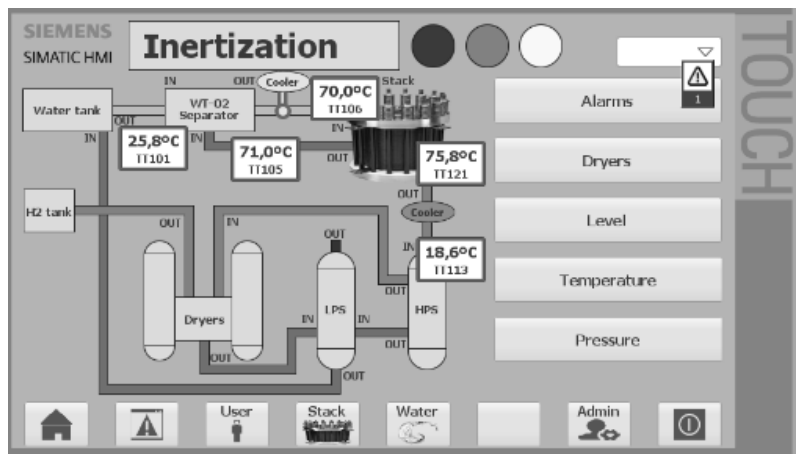

Figure 12b: Hydrogen subsystem. Alarm in the temperature interface.

\section{Conclusions}

Beside hydrogen is a well-known fuel to be used as energy carrier, its production by means green processes based on renewable sources hasn't yet achieved sufficient relevance into the industrial sector. Nevertheless, it is expected that the hydrogen demand increases in the next years especially in distributed generation applications; micro grids based on renewable energy with hydrogen as backup.
Then, it is necessary to focus the efforts towards the development of hydrogen production systems: improving the technology, optimizing the balance of plant and giving control proposals that contribute in the reach of a greater impact of the PEM technology in renewable energies and hydrogen as an energy carrier in power systems.

In this paper, it has been designed the balance of plant of a medium-size PEM electrolyzer and it has been implemented the control system, taking into account operating variables, automation parameters and energy and power efficiency, always under safe conditions.

The PEM electrolyzer requires two main parts that are the water management subsystem and the hydrogen subsystem. In the first part, the control system is responsible for ensuring the water level inside the allowed range, the water flow along the water pipeline, the water temperature and pressure during the production process and the water conductivity to guarantee both the efficiency of the process and the lifetime of the stack.

The hydrogen subsystem need than the controller tracks the levels in the pressure separators (HPS and LPS), acting over the valves that communicates both separators. Additionally, the control system includes the thermal management of the hydrogen flow, the pressure control along the hydrogen flow line and the control the PSA sequence in the final drying stage.

To validate the developed control logic, both subsystems, water and hydrogen, have been simulated. The results show that water and hydrogen subsystems works inside proper operating conditions and in case of warning or sensor malfunctioning, the electrolyzer is stopped through an inertization phase.

\section{Acknowledgment}

This work is a contribution of the Projects DPI201785540-R and UNHU15-CE-3264 supported by the Spanish Ministry of Economy and Competitiveness and by the European Union Regional Development Fund. Additionally, this work is part of the collaborations between the Research Group TEP-192of the University of Huelva, supported by the European Social Fund (ESF) and Junta de Andalucía and Ariema Energía y Mediambiente S.L. 


\section{References}

[1] Daher S de F D. A multicriteria model for selecting renewable energy systems in a distributed generation context. In 2014 IEEE International Conference on Systems, Man, and Cybernetics (SMC), 2014, pp. 625-629. doi: 10.1109/SMC.2014.6973978

[2] Goel S, Sharma R. Performance evaluation of stand alone, grid connected and hybrid renewable energy systems for rural application: A comparative review. Renew. Sustain. Energy Rev., vol. 78, pp. 1378-1389, Oct. 2017. doi: 10.1016/j.rser.2017.05.200

[3] Andujar J M, Segura F, Dominguez T. Study of a renewable energy sources-based smart grid. requirements, targets and solutions. In $20163 \mathrm{rd}$ Conference on Power Engineering and Renewable Energy (ICPERE), 2016, pp. 45-50. doi: 10.1109/ICPERE.2016.7904849

[4] Gandia L M, Arzamendi G, Diéguez PM. Renewable hydrogen technologies: production, purification, storage, applications and safety. Elsevier Science, 2013.

[5] Ayers K E, et al.Research Advances towards Low Cost, High Efficiency PEM Electrolysis. In ECS Transactions, 2010, vol. 33, no. 1, pp. 3-15. doi: 10.1149/1.3484496

[6] Manabe A, et al. Basic study of alkaline water electrolysis. Electrochim. Acta, vol. 100, pp. 249-256, 2013. doi: 10.1016/j.electacta.2012.12.105

[7] Zou H, Chen J, Fang Y, Ding J, Peng W, Liu R. A dualelectrolyte based air-breathing regenerative microfluidic fuel cell with $1.76 \mathrm{~V}$ open-circuit-voltage and $0.74 \mathrm{~V}$ water-splitting voltage. Nano Energy, vol. 27, pp. 619-626, Sep. 2016. doi: 10.1016/J.NANOEN.2016.07.036

[8] Bordons C, García-Torres F, Valverde L. Gestión Óptima de la Energía en Microrredes con Generación Renovable. Rev. Iberoam. Automática e Informática Ind. RIAI, vol. 12, no. 2, pp. 117-132, Apr. 2015. doi: 10.1016/J.RIAI.2015.03.001

[9] Carmo M, Fritz D L, Mergel J, Stolten D. A comprehensive review on PEM water electrolysis. International Journal of Hydrogen Energy, vol. 38, no. 12. pp. 4901-4934, 2013. doi: 10.1016/j.ijhydene.2013.01.151
[10] Vivas F J, De las Heras A, Segura F, Andújar J M. A review of energy management strategies for renewable hybrid energy systems with hydrogen backup. Renew. Sustain. Energy Rev., vol. 82, 2018. doi: 10.1016/j.rser.2017.09.014

[11] Medina P, Santarelli M. Analysis of water transport in a high pressure PEM electrolyzer. Int. J. Hydrogen Energy, vol. 35, no. 11, pp. 5173-5186, Jun. 2010. doi: 10.1016/J.IJHYDENE.2010.02.130

[12] Andolfatto F, Durand F, Michas A, Millet P, Stevens P. Solid polymer electrolyte water electrolysis: electrocatalysis and long-term stability. Int. J. Hydrogen Energy, vol. 19, no. 5, pp. 421-427, May 1994. doi: 10.1016/0360-3199(94)90018-3

[13] Ruuskanen V, et al. Design and implementation of a power-hardware-in-loop simulator for water electrolysis emulation. Renew. Energy, vol. 119, pp. 106-115, Apr. 2018. doi: 10.1016/J.RENENE.2017.11.088

[14] Sanchez V M, Barbosa R, Arriaga LG, Ramirez J M. Real time control of air feed system in a PEM fuel cell by means of an adaptive neural-network. Int. $J$. Hydrogen Energy, vol. 39, no. 29, pp. 16750-16762, Oct. 2014. doi: 10.1016/J.IJHYDENE.2014.05.153.

[15] Agbli K S, Péra MC, Hissel D, Rallières O, Turpin C, Doumbia I. Multiphysics simulation of a PEM electrolyser: Energetic Macroscopic Representation approach. Int. J. Hydrogen Energy, vol. 36, no. 2, pp. 1382-1398, Jan. 2011doi: 10.1016/J.IJHYDENE.2010.10.069

[16] Awasthi A, Scott K, Basu S. Dynamic modeling and simulation of a proton exchange membrane electrolyzer for hydrogen production. Int. J. Hydrogen Energy, vol. 36, no. 22, pp. 14779-14786, Nov. 2011. doi: 10.1016/J.IJHYDENE.2011.03.045

[17] Yigit T, Selamet O F. Mathematical modeling and dynamic Simulink simulation of high-pressure PEM electrolyzer system. Int. J. Hydrogen Energy, vol. 41, no. 32, pp. 13901-13914, Aug. 2016. doi: 10.1016/J.IJHYDENE.2016.06.022

[18] de las Heras A, Vivas F J, Segura F, Andújar J M. How the BoP configuration affects the performance in an aircooled polymer electrolyte fuel cell. Keys to design the best configuration. Int. J. Hydrogen Energy, vol. 42, no. 17, pp. 12841-12855, Apr. 2017. doi: 10.1016/J.IJHYDENE.2016.11.051 